\title{
Cerebrolysin for the Treatment of Aneurysmal Subarachnoid Hemorrhage in Adults: A Retrospective Chart Review
}

\author{
Yung Ki Park · Hyeong-Joong Yi · Kyu-Sun Choi · Young-Jun Lee • \\ Dong-Won Kim · Sae Min Kwon
}

Received: July 10, 2018/Published online: November 92018

(C) The Author(s) 2018, corrected publication 2019

\begin{abstract}
Introduction: Cerebrolysin is a neuroprotective drug used in the treatment of acute ischemic stroke. To our knowledge, this drug has never been evaluated in patients with aneurysmal subarachnoid hemorrhage (SAH). The aim of this study was to evaluate the effect of Cerebrolysin in patients with aneurysmal SAH.
\end{abstract}

Enhanced Digital Features To view enhanced digital features for this article go to https://doi.org/10.6084/ m9.figshare.7275347.

Y. K. Park

Department of Neurosurgery, Seoul St. Mary's Hospital, The Catholic University of Korea, Seoul, Republic of Korea

H.-J. Yi $(\varangle) \cdot$ K.-S. Choi

Department of Neurosurgery, Hanyang University

Medical Center, Seoul, Republic of Korea

e-mail: hjyi8499@hanyang.ac.kr

Y.-J. Lee

Department of Radiology, Hanyang University

Medical Center, Seoul, Republic of Korea

D.-W. Kim

Department of Anesthesiology and Pain Medicine, Hanyang University Medical Center, Seoul, Republic of Korea

\section{S. M. Kwon}

Department of Neurosurgery, University of Ulsan College of Medicine, Asan Medical Center, Seoul, Republic of Korea
Methods: Aneurysmal SAH patients who had their aneurysm obliterated at our institution from 2007 to 2016 were retrospectively studied. Patients received Cerebrolysin treatment or standard care only (control group). Subgroup analyses were performed according to Hunt and Hess grade (good grade $\leq 2, N=216$; poor grade $\geq 3, N=246$ ) and treatment procedure (clip or coil).

Results: In good-grade patients $(N=216)$, clinical outcomes and mortality did not differ significantly between the control and Cerebrolysin groups. In poor-grade patients $(N=246)$, the mortality rate was significantly lower in the Cerebrolysin group (8.7\%) than in the control group $(25.4 \%, p=0.006)$. In patients who received microsurgical clipping $(N=328)$, the mortality rate was significantly lower in the Cerebrolysin group (7.3\%) than in the control group $(18.5 \%, p=0.016)$.

Conclusion: Cerebrolysin injection during the acute period of SAH appeared to reduce the mortality rate, especially in poor-grade patients. This study suggests the potential of Cerebrolysin for treating aneurysmal SAH. Further studies are needed to confirm our results.

Keywords: Aneurysmal subarachnoid hemorrhage; Brain hemorrhage; Cerebrolysin; Cerebrovascular disorders; Neuroprotective agents; Mortality 


\section{INTRODUCTION}

Aneurysmal subarachnoid hemorrhage (SAH) is a devastating disease with high morbidity and mortality. However, treatment remains insufficient, and drugs to improve patient outcomes are not well established. SAH is a complex pathology and involves vasospasm, acute or chronic hydrocephalus, systemic inflammation, and stressful treatments including surgical procedures. Many agents such as clazosentan, simvastatin, and magnesium sulfate have been assessed in large clinical studies; however, in clinical practice the effects have been disappointing [1-3].

Cerebrolysin (EVER Neuro Pharma ${ }^{\mathrm{TM}}$ ) is a neuropeptide preparation that mimics the action of endogenous neurotrophic factors in brain protection and recovery. It has been shown to be effective against excitotoxicity, inhibits free radical formation, has neurotrophic activity, improves cellular survival, and stimulates sprouting, synaptogenesis, and neurogenesis [4-9]. Several clinical studies have shown beneficial effects of Cerebrolysin in stroke, dementia, and traumatic brain injury [10-13]. A recent meta-analysis of nine randomized, double-blind, placebo-controlled stroke studies with 1879 patients confirmed the early beneficial effect of Cerebrolysin on global neurologic deficits [14]. Recently, Cerebrolysin has been successfully tested for its neurorecovery potential in patients with moderate-to-severe strokes with treatment initiation in the acute and subacute phase $[11,15,16]$.

This study investigated potential benefits of Cerebrolysin in patients with aneurysmal SAH. To our knowledge, no such studies have been performed with Cerebrolysin before.

\section{METHODS}

\section{Study Design}

We retrospectively reviewed medical charts from patients with SAH who underwent aneurysmal occlusion at our institution between January 2007 and December 2016. There was no significant change in surgical or endovascular instruments or treatment protocols during this period. The inclusion criteria were: (1) both sexes 18-85 years old, (2) having $\mathrm{SAH}$ within $48 \mathrm{~h}$ before admission, and (3) aneurysm obliterated with either clip or coil within $72 \mathrm{~h}$ after SAH. Exclusion criteria were early death within $72 \mathrm{~h}$ of admission, procedural complication, patients who discontinued Cerebrolysin treatment within 3 days, previous stroke or neurologic deficits, mental disability, psychologic disorders, or lost to follow-up within 3 months. All procedures performed in this study were in accordance with the ethical standards of the institution and with the 1964 Helsinki Declaration and its later amendments. For this type of study, formal consent is not required. This study is not registered as a clinical trial because of its retrospective design. This study was approved by the institutional review board at the author's institute (HYUH IRB 2017-10-007-001).

\section{Treatment Groups}

Patients received Cerebrolysin treatment or standard care only (control group). Cerebrolysin was administered at daily doses of $30 \mathrm{ml}$ for at least 3 days. Cerebrolysin was diluted in $1000 \mathrm{ml}$ normal saline and was administered as a slow intravenous infusion over $24 \mathrm{~h}$. Treatment was initiated within $48 \mathrm{~h}$ after SAH.

\section{Data Collection}

Demographic information, radiologic findings, treatment, and clinical parameters were reviewed. These data included sex, age, hypertension, diabetes mellitus, treatment (clip or coil), and circulation (anterior or posterior). The Glasgow Coma Scale (GCS) and Hunt and Hess grade [17] were used to record each patient's initial condition. The modified Fisher Scale, concomitant intracerebral hemorrhage (ICH), SAH sum score [18], and intraventricular hemorrhage (IVH) sum score [19] were recorded to evaluate initial radiologic findings. SAH sum score (0-30) was calculated as the mean of the Hijdra score, which is the sum of the amount of blood in ten cistern or fissure points ( $0-3$ each). 
Table 1 Demographic features, radiologic findings, and clinical outcomes in total SAH patients

\begin{tabular}{|c|c|c|c|}
\hline & Control $(N=328)$ & Cerebrolysin $(N=134)$ & $p$ value \\
\hline Female & $221(67.4 \%)$ & $84(62.7 \%)$ & 0.391 \\
\hline Age (years) & $55.0[47.0 ; 66.0]$ & $56.0[48.0 ; 62.0]$ & 0.876 \\
\hline \multicolumn{4}{|l|}{ Operation type } \\
\hline Clip & $232(70.7 \%)$ & $96(71.6 \%)$ & \multirow[t]{2}{*}{0.934} \\
\hline Coil & $96(29.3 \%)$ & $38(28.4 \%)$ & \\
\hline \multicolumn{4}{|l|}{ Circulation } \\
\hline Anterior & $294(89.6 \%)$ & $124(92.5 \%)$ & \multirow[t]{2}{*}{0.430} \\
\hline Posterior & $34(10.4 \%)$ & $10(7.5 \%)$ & \\
\hline \multicolumn{4}{|l|}{ Hunt and Hess grade } \\
\hline Grade 1 & $25(7.6 \%)$ & $9(6.7 \%)$ & \multirow[t]{5}{*}{0.567} \\
\hline Grade 2 & $126(38.4 \%)$ & $56(41.8 \%)$ & \\
\hline Grade 3 & $93(28.4 \%)$ & $34(25.4 \%)$ & \\
\hline Grade 4 & $79(24.1 \%)$ & $30(22.4 \%)$ & \\
\hline Grade 5 & $5(1.5 \%)$ & $5(3.7 \%)$ & \\
\hline \multicolumn{4}{|l|}{ Modified Fisher scale } \\
\hline 1 & $31(9.5 \%)$ & $12(9.0 \%)$ & \multirow[t]{4}{*}{0.096} \\
\hline 2 & $5(1.5 \%)$ & $5(3.7 \%)$ & \\
\hline 3 & $119(36.3 \%)$ & $35(26.1 \%)$ & \\
\hline 4 & $173(52.7 \%)$ & $82(61.2 \%)$ & \\
\hline GCS score & $13.0[9.5 ; 15.0]$ & $14.0[8.0 ; 15.0]$ & 0.964 \\
\hline Concomitant ICH & $94(28.7 \%)$ & $47(35.1 \%)$ & 0.212 \\
\hline Bicaudate index & $17.6[14.9 ; 20.2]$ & $15.7[13.9 ; 18.3]$ & $<0.001^{*}$ \\
\hline SAH sum score & $19.0[11.0 ; 26.0]$ & $25.0[14.0 ; 28.0]$ & $0.002^{*}$ \\
\hline IVH sum score & $1.0[0.0 ; 4.0]$ & $1.0[0.0 ; 3.0]$ & 0.375 \\
\hline Smoking & $86(26.2 \%)$ & $39(29.1 \%)$ & 0.604 \\
\hline Hypertension & $126(38.4 \%)$ & $49(36.6 \%)$ & 0.790 \\
\hline Diabetes mellitus & $19(5.8 \%)$ & $14(10.4 \%)$ & 0.118 \\
\hline Permanent shunt operation & $47(14.3 \%)$ & $21(15.7 \%)$ & 0.822 \\
\hline Angiographic vasospasm & $100(30.5 \%)$ & $55(41.0 \%)$ & $0.038^{*}$ \\
\hline Delayed cerebral ischemia & $43(13.1 \%)$ & $23(17.2 \%)$ & 0.325 \\
\hline Days of Cerebrolysin & $0.0[0.0 ; 0.0]$ & $13.0[10.0 ; 20.0]$ & \\
\hline Length of stay (days) & $24.0[16.0 ; 47.0]$ & $22.0[15.0 ; 46.0]$ & 0.289 \\
\hline $\mathrm{mRS}$ & $2.0[1.0 ; 5.0]$ & $2.0[1.0 ; 5.0]$ & 0.398 \\
\hline
\end{tabular}


Table 1 continued

\begin{tabular}{llll}
\hline & Control $(\boldsymbol{N}=\mathbf{3 2 8})$ & Cerebrolysin $(\boldsymbol{N}=\mathbf{1 3 4})$ & $\boldsymbol{p}$ value \\
\hline Clinical outcome & & $70(52.2 \%)$ & 0.636 \\
Favorable (mRS 0-2) & $181(55.2 \%)$ & $64(47.8 \%)$ & $0.031^{*}$ \\
Unfavorable (mRS 3-6) & $147(44.8 \%)$ & $12(9.0 \%)$ & \\
Mortality & $57(17.4 \%)$ &
\end{tabular}

$S A H$ subarachnoid hemorrhage, GCS Glasgow Coma Scale, $I C H$ intracerebral hemorrhage, $I V H$ intraventricular hemorrhage, $m R S$ modified Rankin scale

${ }^{*}$ Indicates statistical significance $(p<0.05)$

IVH sum score (0-12) was calculated as the mean Graeb score, which is the sum of the score for each ventricle (lateral ventricles, 0-4; third ventricle, 0-2; fourth ventricle, 0-2). Chronic hydrocephalus leading permanent shunt operation was reviewed. Angiographic vasospasm was defined as $>50 \%$ decrease of the intracranial artery diameter by imaging or diagnostic angiography. Delayed cerebral ischemia was defined as the occurrence of focal neurologic impairment or a decrease of at least two points on the GCS score or one of its individual components lasting at least $1 \mathrm{~h}$ that could not be attributed to other causes [20]. A blinded neuroradiologist at our institution recorded radiologic findings. The modified Rankin Scale (mRS) was used to evaluate clinical outcome 3 months after SAH [21]. Mortality was defined as inhospital death later than $72 \mathrm{~h}$ after SAH ictus.

\section{Treatment of SAH Patients}

All patients had their aneurysm obliterated by either microscopic aneurysm neck clipping or endovascular coil embolization with or without stent assist. The therapy was chosen by the neurovascular team based on age, mental status, aneurysm location, size, and neck/dome presentation. All patients with posterior circulation including the vertebral and basilar arteries were treated with endovascular coil embolization. Blood pressure and glucose were managed strictly with close observation according to our institution's protocol. Patients in the Cerebrolysin group were not treated any differently from the control group except for Cerebrolysin.

\section{Patient Group Classification}

Subgroup analyses were performed according to the severity of subarachnoid hemorrhage classified by the Hunt and Hess grade (good grade $\leq 2, \quad N=216$; poor grade $\geq 3, N=246$ ) and treatment procedure (clipping, coiling).

\section{Statistical Analysis}

Non-parametric data were compared with chisquare and Fisher's exact tests. Parametric variables with a normal distribution were compared by independent $t$ test and those without a normal distribution by Mann-Whitney $U$ test. Descriptive summaries were reported as mean ( \pm standard deviation) for continuous variables with normal distribution, median [interquartile range (IQR)] for continuous variables without normal distribution, and frequency (percentage) for categorical variables. All data were analyzed with $\mathrm{R}$ version 3.3.2 (https://www.rproject.org/; R Foundation for Statistical Computing, Vienna, Austria).

\section{RESULTS}

\section{Total Aneurysmal SAH Patients}

Overall, 548 aneurysmal SAH patients were treated at our institution from 2007 to 2016; of these, 462 patients were included in this study. Patients were excluded because of early death within $72 \mathrm{~h}(N=15)$, procedural complication $(N=8)$, previous neurologic deficits $(N=9)$, 
Table 2 Demographic features, radiologic findings, and clinical outcomes in good-grade SAH patients

\begin{tabular}{|c|c|c|c|}
\hline & Control $(N=151)$ & Cerebrolysin $(N=65)$ & $p$ value \\
\hline Female & $104(68.9 \%)$ & $39(60.0 \%)$ & 0.268 \\
\hline Age & $53.0[44.0 ; 64.0]$ & $55.0[47.0 ; 59.0]$ & 0.581 \\
\hline \multicolumn{4}{|l|}{ Operation type } \\
\hline Clip & $103(68.2 \%)$ & $45(69.2 \%)$ & \multirow[t]{2}{*}{1.000} \\
\hline Coil & $48(31.8 \%)$ & $20(30.8 \%)$ & \\
\hline \multicolumn{4}{|l|}{ Circulation } \\
\hline Anterior & $141(93.4 \%)$ & $61(93.8 \%)$ & \multirow[t]{2}{*}{1.000} \\
\hline Posterior & $10(6.6 \%)$ & $4(6.2 \%)$ & \\
\hline \multicolumn{4}{|l|}{ Hunt and Hess grade } \\
\hline Grade 1 & $25(16.6 \%)$ & $9(13.8 \%)$ & \multirow[t]{2}{*}{0.766} \\
\hline Grade 2 & $126(83.4 \%)$ & $56(86.2 \%)$ & \\
\hline \multicolumn{4}{|l|}{ Modified Fisher scale } \\
\hline 1 & $27(17.9 \%)$ & $9(13.8 \%)$ & \multirow[t]{4}{*}{0.357} \\
\hline 2 & $2(1.3 \%)$ & $2(3.1 \%)$ & \\
\hline 3 & $66(43.7 \%)$ & $23(35.4 \%)$ & \\
\hline 4 & $56(37.1 \%)$ & $31(47.7 \%)$ & \\
\hline GCS score & $15.0[15.0 ; 15.0]$ & $15.0[15.0 ; 15.0]$ & 0.761 \\
\hline Concomitant ICH & $23(15.2 \%)$ & $14(21.5 \%)$ & 0.352 \\
\hline Bicaudate index & $16.7[14.5 ; 19.4]$ & $15.8[14.1 ; 17.7]$ & $0.043^{*}$ \\
\hline SAH sum score & $14.0[8.0 ; 22.0]$ & $17.0[10.0 ; 27.0]$ & 0.051 \\
\hline IVH sum score & $0.0[0.0 ; 1.0]$ & $1.0[0.0 ; 2.0]$ & 0.106 \\
\hline Smoking & $40(26.5 \%)$ & $21(32.3 \%)$ & 0.480 \\
\hline Hypertension & $46(30.5 \%)$ & $24(36.9 \%)$ & 0.440 \\
\hline Diabetes mellitus & $5(3.3 \%)$ & $7(10.8 \%)$ & 0.061 \\
\hline Permanent shunt & $15(9.9 \%)$ & $5(7.7 \%)$ & 0.791 \\
\hline Angiographic vasospasm & $49(32.5 \%)$ & $19(29.2 \%)$ & 0.758 \\
\hline Delayed cerebral ischemia & $18(11.9 \%)$ & $7(10.8 \%)$ & 0.991 \\
\hline Days of Cerebrolysin & $0.0[0.0 ; 0.0]$ & $12.0[9.0 ; 17.0]$ & \\
\hline Length of stay (days) & $22.0[17.0 ; 34.0]$ & $18.0[15.0 ; 26.0]$ & $0.015^{*}$ \\
\hline $\mathrm{mRS}$ & $1.0[1.0 ; 2.0]$ & $1.0[0.0 ; 2.0]$ & 0.145 \\
\hline \multicolumn{4}{|l|}{ Clinical outcome } \\
\hline Favorable (mRS 0-2) & $119(78.8 \%)$ & $50(76.9 \%)$ & \multirow[t]{2}{*}{0.898} \\
\hline Unfavorable (mRS 3-6) & $32(21.2 \%)$ & $15(23.1 \%)$ & \\
\hline
\end{tabular}


Table 2 continued

\begin{tabular}{llll}
\hline & Control $(\boldsymbol{N}=\mathbf{1 5 1})$ & Cerebrolysin $(\boldsymbol{N}=\mathbf{6 5})$ & $\boldsymbol{p}$ value \\
\hline Mortality & $12(7.9 \%)$ & $6(9.2 \%)$ & 0.964 \\
\hline
\end{tabular}

$S A H$ subarachnoid hemorrhage, GCS Glasgow Coma Scale, $I C H$ intracerebral hemorrhage, $I V H$ intraventricular hemorrhage, $m R S$ modified Rankin Scale

${ }^{*}$ Indicates statistical significance $(p<0.05)$

discontinuation of Cerebrolysin within 3 days $(N=19)$, and follow-up loss within 3 months $(N=35)$. Of 462 patients with SAH, 134 were included in the Cerebrolysin group and 328 in the control group. Demographic features, radiologic findings, and clinical outcomes are presented in Table 1. Median treatment duration with Cerebrolysin was 13 [10.0; 20.0] days. Baseline parameters did not differ between groups except for the initial bicaudate index, which was higher in the control group (median; 17.6 vs. 15.7, $p<0.001$ ) and for the SAH sum score, which was higher in the Cerebrolysin group (median; 25.0 vs. 19.0, $p=0.002$ ). Angiographic vasospasm occurred more often in the Cerebrolysin group than in the control group (41.0 vs. $30.5, p=0.038$ ). Three months after SAH, patients of both groups had a median mRS score of 2; the mortality rate was significantly higher in the control group (17.4\%) than in the Cerebrolysin group $(9.0 \%, p=0.031)$.

\section{Good-Grade SAH Patients (Hunt and Hess Grade $\leq$ 2)}

Of 216 patients with Hunt and Hess grade $\leq 2$, 65 were included in the Cerebrolysin group and 151 in the control group. Demographic features, radiologic findings, and clinical outcomes are shown in Table 2. Median treatment duration with Cerebrolysin was 12 [9.0; 17.0] days. Baseline parameters did not differ between groups except for the initial bicaudate index, which was significantly higher in the control group (median; 16.7 vs. $15.8, p=0.043$ ). Length of stay was significantly reduced by 4 days in the Cerebrolysin group, with statistical significance ( 22 vs. 18 median days, $p=0.015$ ). Three months after $\mathrm{SAH}$, patients of both groups had a median mRS score of 1 ; no significant group differences were reported for mortality.

\section{Poor-Grade SAH Patients (Hunt and Hess Grade $\geq 3$ )}

Of 246 patients with Hunt and Hess grade $\geq 3$, 69 were included in the Cerebrolysin group and 177 in the control group. Demographic features, radiologic findings, and clinical outcomes are shown in Table 3. Median treatment duration with Cerebrolysin was 14 [12.0; 21.0] days. Baseline parameters did not differ between groups except for the initial bicaudate index, which was significantly higher in the control group (median; 18.1 vs. $15.7, p=0.001$ ) and for the SAH sum score, which was significantly higher in the Cerebrolysin group (median; 27.0 vs. 25.0, $p=0.001$ ). Angiographic vasospasm occurred more often in the Cerebrolysin group compared with the control group $(52.2 \%$ vs. $28.8 \%, p=0.001)$. Three months after SAH, patients of both groups had a median mRS score of 4 ; the mortality rate was significantly higher in the control group (25.4\%) than in the Cerebrolysin group $(8.7 \%, p=0.006)$.

\section{Clip and Coil Patients}

Of 462 patients, 328 (71.0\%) had microsurgical aneurysm neck clipping and 134 (29.0\%) had endovascular coil embolization. Demographic features, radiologic findings, and clinical outcomes are shown in Table 4 . In clip patients, the initial bicaudate index was significantly higher in the control group (median; 17.5 vs. 15.5, $p<0.001$ ) and the SAH sum score was significantly higher in the Cerebrolysin group (median; 25.0 vs. 19.0, $p=0.007)$. Angiographic 
Table 3 Demographic features, radiologic findings, and clinical outcomes in poor-grade SAH patients

\begin{tabular}{|c|c|c|c|}
\hline & Control $(N=177)$ & Cerebrolysin $(N=69)$ & $p$ value \\
\hline Female sex & $117(66.1 \%)$ & $45(65.2 \%)$ & 1.000 \\
\hline Age & $56.0[49.0 ; 68.0]$ & $56.0[50.0 ; 65.0]$ & 0.599 \\
\hline \multicolumn{4}{|l|}{ Operation type } \\
\hline Clip & $129(72.9 \%)$ & $51(73.9 \%)$ & \multirow[t]{2}{*}{0.997} \\
\hline Coil & $48(27.1 \%)$ & $18(26.1 \%)$ & \\
\hline \multicolumn{4}{|l|}{ Circulation } \\
\hline Anterior & $153(86.4 \%)$ & $63(91.3 \%)$ & \multirow[t]{2}{*}{0.406} \\
\hline Posterior & $24(13.6 \%)$ & $6(8.7 \%)$ & \\
\hline \multicolumn{4}{|l|}{ Hunt and Hess grade } \\
\hline Grade 3 & $93(52.5 \%)$ & $34(49.3 \%)$ & \multirow[t]{3}{*}{0.286} \\
\hline Grade 4 & $79(44.6 \%)$ & $30(43.5 \%)$ & \\
\hline Grade 5 & $5(2.8 \%)$ & $5(7.2 \%)$ & \\
\hline \multicolumn{4}{|l|}{ Modified Fisher scale } \\
\hline 1 & $4(2.3 \%)$ & $3(4.3 \%)$ & \multirow[t]{4}{*}{0.133} \\
\hline 2 & $3(1.7 \%)$ & $3(4.3 \%)$ & \\
\hline 3 & $53(29.9 \%)$ & $12(17.4 \%)$ & \\
\hline 4 & $117(66.1 \%)$ & $51(73.9 \%)$ & \\
\hline GCS score & $11.0[6.0 ; 13.0]$ & $8.0[6.0 ; 13.0]$ & 0.447 \\
\hline Concomitant ICH & $71(40.1 \%)$ & $33(47.8 \%)$ & 0.339 \\
\hline Bicaudate index & $18.1[15.3 ; 21.3]$ & $15.7[13.7 ; 18.8]$ & $0.001^{*}$ \\
\hline SAH sum score & $25.0[14.0 ; 27.0]$ & $27.0[23.0 ; 29.0]$ & $0.001^{*}$ \\
\hline IVH sum score & $2.0[0.0 ; 5.0]$ & $2.0[1.0 ; 4.0]$ & 0.994 \\
\hline Smoking & $46(26.0 \%)$ & $18(26.1 \%)$ & 1.000 \\
\hline Hypertension & $80(45.2 \%)$ & $25(36.2 \%)$ & 0.257 \\
\hline Diabetes mellitus & $14(7.9 \%)$ & $7(10.1 \%)$ & 0.757 \\
\hline Permanent shunt & $32(18.1 \%)$ & $16(23.2 \%)$ & 0.466 \\
\hline Angiographic vasospasm & $51(28.8 \%)$ & $36(52.2 \%)$ & 0.001 \\
\hline Delayed cerebral ischemia & $25(14.1 \%)$ & $16(23.2 \%)$ & 0.128 \\
\hline Days of Cerebrolysin & $0.0[0.0 ; 0.0]$ & $14.0[12.0 ; 21.0]$ & \\
\hline Length of stay (days) & $30.0[15.0 ; 67.0]$ & $33.0[16.0 ; 70.0]$ & 0.547 \\
\hline $\mathrm{mRS}$ & $4.0[2.0 ; 6.0]$ & $4.0[2.0 ; 5.0]$ & 0.525 \\
\hline Clinical outcome & & & \\
\hline
\end{tabular}


Table 3 continued

\begin{tabular}{llll}
\hline & Control $(\boldsymbol{N}=\mathbf{1 7 7})$ & Cerebrolysin $(\boldsymbol{N}=\mathbf{6 9})$ & $\boldsymbol{p}$ value \\
\hline Favorable (mRS 0-2) & $62(35.0 \%)$ & $20(29.0 \%)$ & 0.452 \\
Unfavorable (mRS 3-6) & $115(65.0 \%)$ & $49(71.0 \%)$ & $0.006^{*}$ \\
Mortality & $45(25.4 \%)$ & $6(8.7 \%)$ & \\
\hline
\end{tabular}

$S A H$ subarachnoid hemorrhage, GCS Glasgow Coma Scale, $I C H$ intracerebral hemorrhage, $I V H$ intraventricular hemorrhage, $m R S$ modified Rankin Scale

*Indicates statistical significance $(p<0.05)$

vasospasm developed more often in the Cerebrolysin group $(33.6 \%$ vs. $47.9 \%, p=0.021)$, and delayed cerebral ischemia was not statistically different between groups $(12.5 \%$ vs. $20.8 \%, p=0.079)$. Three months after SAH, the mortality rate was significantly lower in the Cerebrolysin group (median; $7.3 \%$ vs. $18.5 \%$, $p=0.016)$. No significant group differences were observed in the mRS. Coil patients did not differ in baseline characteristics or outcome parameters.

\section{Safety}

The median treatment duration with Cerebrolysin was 13.0 days. Table 5 shows the rate of adverse events with severe intensity for both groups. The most common adverse event was pneumonia followed by urinary tract infection, acute renal failure, and myocardial infarction. There was no significant difference between the two groups.

\section{DISCUSSION}

The current study showed excellent outcomes in both study groups and a similar low mortality rate in SAH patients with good grade according to Hunt and Hess. In contrast, in patients with poor grade, the mortality rate was significantly higher in the control group (25.4\% versus $8.7 \%$ ) than in Cerebrolysin-treated patients. These findings are reminiscent of previous studies showing beneficial effects of Cerebrolysin especially in more severely affected patients of different brain pathologies. The results of the
CASTA trial reported a reduced mortality rate in more severely affected ischemic stroke patients treated with Cerebrolysin [22]. Similarly, Khalili et al. showed that Cerebrolysin is associated with improved functional recovery, decreased mortality rate, and better outcome in patients with severe disability after traumatic brain injury [23].

Of note, our study showed a lower mortality rate in the Cerebrolysin group in patients with microsurgical clipping but not in patients with endovascular coiling. This might be because surgical clipping was more frequently performed in patients with higher Hunt and Hess grades and in patients with concomitant ICH because of the advantage of evacuating the hematoma simultaneously. Although angiographic vasospasm and delayed cerebral ischemia occurred more often in poor-grade $\mathrm{SAH}$ patients treated with Cerebrolysin, the mortality rate was higher in the control group.

Inhibition of brain edema seems to be a key mechanism for lowering the mortality rate in the acute phase after SAH. Maintaining the blood-brain barrier integrity can reduce vasogenic edema, and anti-inflammatory effects contribute to decreased cytotoxic edema [24]. An intracranial hemorrhage rat model showed that Cerebrolysin inhibited brain edema and the inflammatory response and protected the integrity of the blood-brain barrier [10]. In a mouse stroke model study, Cerebrolysin inhibited the effect of proinflammatory mediators such as TNF- $\alpha$, IL-1 $\beta$, IL- 6 , and NF- $\kappa B[21,22]$. Unfortunately, we could not assess the effect of Cerebrolysin on brain edema formation in the 
Table 4 Demographic features, radiologic findings, and clinical outcomes by operation type (clip versus coil)

\begin{tabular}{|c|c|c|c|c|c|c|}
\hline & \multicolumn{3}{|l|}{ Clip $(N=328)$} & \multicolumn{3}{|l|}{ Coil $(N=134)$} \\
\hline & $\begin{array}{l}\text { Control } \\
(N=232)\end{array}$ & $\begin{array}{l}\text { Cerebrolysin } \\
(N=96)\end{array}$ & $p$ & $\begin{array}{l}\text { Control } \\
(N=96)\end{array}$ & $\begin{array}{l}\text { Cerebrolysin } \\
(N=38)\end{array}$ & $p$ \\
\hline Female & $155(66.8 \%)$ & $59(61.5 \%)$ & 0.424 & $66(68.8 \%)$ & $25(65.8 \%)$ & 0.900 \\
\hline Age & $55.0[48.0 ; 66.0]$ & $55.0[49.0 ; 61.5]$ & 0.601 & $55.1 \pm 13.3$ & $56.4 \pm 14.4$ & 0.639 \\
\hline \multicolumn{7}{|l|}{ Circulation } \\
\hline Anterior & $232(100.0 \%)$ & $96(100.0 \%)$ & & $62(64.6 \%)$ & $28(73.7 \%)$ & 0.420 \\
\hline Posterior & $0(0.0 \%)$ & $0(0.0 \%)$ & & $34(35.4 \%)$ & $10(26.3 \%)$ & \\
\hline Hunt and Hess grade & $3.0[2.0 ; 4.0]$ & $3.0[2.0 ; 4.0]$ & 0.778 & $2.5[2.0 ; 3.0]$ & $2.0[2.0 ; 3.0]$ & 0.832 \\
\hline \multicolumn{7}{|l|}{ Modified Fisher scale } \\
\hline 1 and 2 & $22(9.5 \%)$ & $11(11.5 \%)$ & 0.734 & $14(14.6 \%)$ & $6(15.8 \%)$ & 1.000 \\
\hline 3 and 4 & $210(90.5 \%)$ & $85(88.5 \%)$ & & $82(85.4 \%)$ & $32(84.2 \%)$ & \\
\hline GCS score & $13.0[8.0 ; 15.0]$ & $13.0[7.0 ; 15.0]$ & 0.843 & $\begin{array}{l}14.0[12.0 ; \\
15.0]\end{array}$ & $14.0[9.0 ; 15.0]$ & 0.872 \\
\hline Bicaudate index & $17.5 \pm 3.9$ & $15.5 \pm 3.2$ & $<0.001^{*}$ & $\begin{array}{c}17.8[15.1 ; \\
20.8]\end{array}$ & $16.9[14.8 ; 21.8]$ & 0.706 \\
\hline SAH sum score & $19.0[12.0 ; 26.0]$ & $25.0[14.0 ; 28.0]$ & $0.007^{*}$ & $17.5[8.5 ; 26.0]$ & $23.0[11.0 ; 28.0]$ & 0.102 \\
\hline IVH sum score & $1.0[0.0 ; 4.0]$ & $1.0[0.0 ; 2.0]$ & 0.989 & $1.0[0.0 ; 4.0]$ & $2.0[0.0 ; 6.0]$ & 0.099 \\
\hline Concomitant ICH & $81(34.9 \%)$ & $41(42.7 \%)$ & 0.229 & $13(13.5 \%)$ & $6(15.8 \%)$ & 0.951 \\
\hline Smoking & $66(28.4 \%)$ & $20(20.8 \%)$ & 0.197 & $29(30.2 \%)$ & $10(26.3 \%)$ & 0.813 \\
\hline Hypertension & $82(35.3 \%)$ & $37(38.5 \%)$ & 0.673 & $44(45.8 \%)$ & $12(31.6 \%)$ & 0.189 \\
\hline Diabetes mellitus & $16(6.9 \%)$ & $11(11.5 \%)$ & 0.251 & $3(3.1 \%)$ & $3(7.9 \%)$ & 0.459 \\
\hline Permanent shunt & $38(16.4 \%)$ & $17(17.7 \%)$ & 0.896 & $9(9.4 \%)$ & $4(10.5 \%)$ & 1.000 \\
\hline $\begin{array}{l}\text { Angiographic } \\
\text { vasospasm }\end{array}$ & $78(33.6 \%)$ & $46(47.9 \%)$ & $0.021^{*}$ & $22(22.9 \%)$ & $9(23.7 \%)$ & 1.000 \\
\hline $\begin{array}{l}\text { Delayed cerebral } \\
\text { ischemia }\end{array}$ & $29(12.5 \%)$ & $20(20.8 \%)$ & 0.079 & $14(14.6 \%)$ & $3(7.9 \%)$ & 0.447 \\
\hline Days of Cerebrolysin & & $14.0[11.0 ; 20.5]$ & & & $12.0[8.0 ; 16.0]$ & \\
\hline Length of stay (days) & $27.0[17.0 ; 60.0]$ & $23.0[16.0 ; 50.5]$ & 0.372 & $\begin{array}{l}20.0[15.0 ; \\
33.5]\end{array}$ & $18.0[14.0 ; 32.0]$ & 0.407 \\
\hline mRS score & $2.0[1.0 ; 5.0]$ & $3.0[1.0 ; 5.0]$ & 0.239 & $1.0[1.0 ; 4.0]$ & $2.0[1.0 ; 4.0]$ & 0.743 \\
\hline \multicolumn{7}{|l|}{ Clinical outcome } \\
\hline $\begin{array}{l}\text { Favorable (mRS } \\
0-2)\end{array}$ & $119(51.3 \%)$ & $46(47.9 \%)$ & 0.663 & $62(64.6 \%)$ & $24(63.2 \%)$ & 1.000 \\
\hline $\begin{array}{l}\text { Unfavorable (mRS } \\
3-6)\end{array}$ & $113(48.7 \%)$ & $50(52.1 \%)$ & & $34(35.4 \%)$ & $14(36.8 \%)$ & \\
\hline
\end{tabular}


Table 4 continued

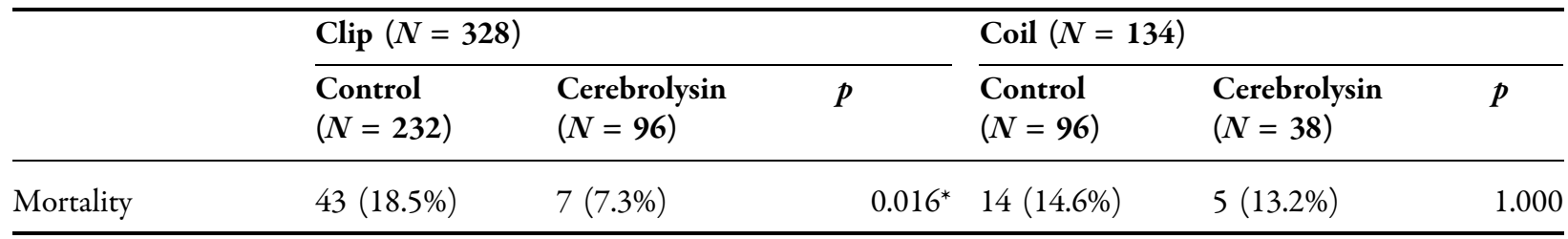

$S A H$ subarachnoid hemorrhage, GCS Glasgow Coma Scale, ICH intracerebral hemorrhage, $I V H$ intraventricular hemorrhage, $m R S$ modified Rankin Scale

*Indicates statistical significance $(p<0.05)$

Table 5 Comparison of adverse events of severe intensity between control and Cerebrolysin groups

\begin{tabular}{lccc}
\hline & Control $(\boldsymbol{N}=\mathbf{3 2 8})$ & Cerebrolysin $(\boldsymbol{N}=\mathbf{1 3 4})$ & $\boldsymbol{p}$ value \\
\hline Pneumonia & $29(8.84 \%)$ & $12(8.96 \%)$ & 1.000 \\
Urinary tract infection & $18(5.49 \%)$ & $8(5.97 \%)$ & 0.986 \\
CSF infection & $3(0.91 \%)$ & $2(1.49 \%)$ & 0.630 \\
Acute renal failure & $17(5.18 \%)$ & $4(2.99 \%)$ & 0.434 \\
Pulmonary embolism & $5(1.52 \%)$ & $1(0.75 \%)$ & 0.678 \\
Acute cholecystitis & $0(0.00 \%)$ & $1(0.75 \%)$ & \\
Myocardial infarction & $14(4.29 \%)$ & $5(3.73 \%)$ & 1.000 \\
Severe adverse event & $82(25.0 \%)$ & $31(23.1 \%)$ & 0.761 \\
\hline
\end{tabular}

CSF cerebrospinal fluid

current study, but it would be interesting to address this in future studies.

One of the limitations of this study was the retrospective design. Accordingly, data from medical records were limited and did not allow precise matching of patients in terms of baseline characteristics. Furthermore, treatment with Cerebrolysin was limited to the acute phase of SAH, and treatment duration varied between 8 and 21 days. For efficacy assessment, no data were available regarding functional disability or cognitive or neuropsychologic outcome. Due to the exclusion of patients who died within $72 \mathrm{~h}(N=15)$, the mortality rate might have been slightly underestimated. However, to our knowledge, this is the largest study that evaluated the effects of Cerebrolysin in aneurysmal SAH patients. Despite the limited level of evidence of this retrospective study, we think that these results are promising and could provide guidance for future randomized studies evaluating the effect of Cerebrolysin on $\mathrm{SAH}$ patients.

\section{CONCLUSIONS}

Hemorrhagic stroke including aneurysmal SAH is a devastating disease causing severe bain damage. This study suggests a potential benefit of Cerebrolysin to reduce the mortality rate in patients with aneurysmal SAH, which should be further evaluated in clinical studies.

\section{ACKNOWLEDGEMENTS}

We thank the participants of this study. 
Funding. No funding or sponsorship was received for this study. EVER Pharmaceuticals funded the article processing charges and open access fee.

Authorship. All named authors meet the International Committee of Medical Journal Editors (ICMJE) criteria for authorship for this article, take responsibility for the integrity of the work as a whole, and have given their approval for this version to be published.

Disclosure. Yung Ki Park, Hyeong-Joong Yi, Kyu-Sun Choi, Young-Jun Lee, Dong-Won Kim, and Sae Min Kwon have nothing to disclose.

Compliance with Ethics Guidelines. All procedures performed in this study were in accordance with the ethical standards of the institution and with the 1964 Helsinki Declaration and its later amendments. For this type of study, formal consent is not required. This study was approved by the institutional review board at the author's institute (HYUH IRB 2017-10-007-001).

Data Availability. The data sets generated during and/or analyzed during the current study are available from the corresponding author on reasonable request.

Open Access. This article is distributed under the terms of the Creative Commons Attribution-NonCommercial 4.0 International License (http://creativecommons.org/licenses/ by-nc/4.0/), which permits any noncommercial use, distribution, and reproduction in any medium, provided you give appropriate credit to the original author(s) and the source, provide a link to the Creative Commons license, and indicate if changes were made.

\section{REFERENCES}

1. Macdonald RL, Higashida RT, Keller E, Mayer SA, Molyneux A, Raabe A, et al. Clazosentan, an endothelin receptor antagonist, in patients with aneurysmal subarachnoid haemorrhage undergoing surgical clipping: a randomised, double-blind, placebo-controlled phase 3 trial (CONSCIOUS-2). Lancet Neurol. 2011;10:618-25.

2. Dorhout Mees SM, Algra A, Vandertop WP, van Kooten F, Kuijsten HAJM, Boiten J, et al. Magnesium for aneurysmal subarachnoid haemorrhage (MASH-2): a randomised placebo-controlled trial. Lancet Lond Engl. 2012;380:44-9.

3. Kirkpatrick PJ, Turner CL, Smith C, Hutchinson PJ, Murray GD, STASH Collaborators. Simvastatin in aneurysmal subarachnoid haemorrhage (STASH): a multicentre randomised phase 3 trial. Lancet Neurol. 2014;13:666-75.

4. Formichi P, Radi E, Battisti C, Di Maio G, Muresanu D, Federico A. Cerebrolysin administration reduces oxidative stress-induced apoptosis in limphocytes from healthy individuals. J Cell Mol Med. 2012;16:2840-3.

5. Gutmann B, Hutter-Paier B, Skofitsch G, Windisch $\mathrm{M}$, Gmeinbauer R. In vitro models of brain ischemia: The peptidergic drug cerebrolysin protects cultured chick cortical neurons from cell death. Neurotox Res. 2002;4:59-65.

6. Masliah E, Díez-Tejedor E. The pharmacology of neurotrophic treatment with cerebrolysin: brain protection and repair to counteract pathologies of acute and chronic neurological disorders. Drugs Today. 2012;48:3-24.

7. Sharma HS, Zimmermann-Meinzingen S, Johanson CE. Cerebrolysin reduces blood-cerebrospinal fluid barrier permeability change, brain pathology, and functional deficits following traumatic brain injury in the rat. Ann N Y Acad Sci. 2010;1199:125-37.

8. Zhang C, Chopp M, Cui Y, Wang L, Zhang R, Zhang $\mathrm{L}$, et al. Cerebrolysin enhances neurogenesis in the ischemic brain and improves functional outcome after stroke. J Neurosci Res. 2010;88:3275-81.

9. Zhang L, Chopp M, Meier DH, Winter S, Wang L, Szalad A, et al. Sonic hedgehog signaling pathway mediates cerebrolysin-improved neurological function after stroke. Stroke. 2013;44:1965-72.

10. Bornstein N, Poon WS. Accelerated recovery from acute brain injuries: clinical efficacy of neurotrophic treatment in stroke and traumatic brain injuries. Drugs Today. 2012;48:43-61.

11. Guekht A, Vester J, Heiss W-D, Hoemberg V, Rahlfs VW, et al. Safety and efficacy of Cerebrolysin in motor function recovery after stroke: a meta-analysis of the CARS trials. Neurol Sci. 2017;38:1761-9.

12. Martinez Sánchez BR, Hernández Hernandez JJ, Franco Del Águila DI, Gryzbowski Gainza E, Paz Ballesteros WC, Herrera Rojas J, et al. An open label, 
one arm study to evaluate the efficacy and safety of cerebrolysin in patients with acute severe ischemic stroke in mexico. Value Health J Int Soc Pharmacoeconomics Outcomes Res. 2015;18:A827-8.

13. Muresanu DF, Ciurea AV, Gorgan RM, Gheorghita E, Florian SI, Stan H, et al. A retrospective, multicenter cohort study evaluating the severity- related effects of cerebrolysin treatment on clinical outcomes in traumatic brain injury. CNS Neurol Disord: Drug Targets. 2015;14:587-99.

14. Brainin M. Cerebrolysin: a multi-target drug for recovery after stroke. Expert Rev Neurother. 2018;18:681-7.

15. Muresanu DF, Heiss W-D, Hoemberg V, Bajenaru O, Popescu CD, Vester JC, et al. Cerebrolysin and recovery after stroke (CARS): a randomized, placebo-controlled, double-blind. Multicenter Trial. Stroke. 2016;47:151-9.

16. Chang WH, Park C, Kim DY, Shin Y-I, Ko M-H, Lee A, et al. Cerebrolysin combined with rehabilitation promotes motor recovery in patients with severe motor impairment after stroke. BMC Neurol. 2016;16:31.

17. Hunt WE, Hess RM. Surgical risk as related to time of intervention in the repair of intracranial aneurysms. J Neurosurg. 1968;28:14-20.

18. Hijdra A, Brouwers PJ, Vermeulen M, van Gijn J. Grading the amount of blood on computed tomograms after subarachnoid hemorrhage. Stroke. 1990;21:1156-61.

19. Graeb DA, Robertson WD, Lapointe JS, Nugent RA, Harrison PB. Computed tomographic diagnosis of intraventricular hemorrhage. Etiology and prognosis. Radiology. 1982;143:91-6.

20. Vergouwen MDI, Vermeulen M, van Gijn J, Rinkel GJE, Wijdicks EF, Muizelaar JP, et al. Definition of delayed cerebral ischemia after aneurysmal subarachnoid hemorrhage as an outcome event in clinical trials and observational studies: proposal of a multidisciplinary research group. Stroke. 2010;41:2391-5.

21. van Swieten JC, Koudstaal PJ, Visser MC, Schouten HJ, van Gijn J. Interobserver agreement for the assessment of handicap in stroke patients. Stroke. 1988;19:604-7.

22. Heiss W-D, Brainin M, Bornstein NM, Tuomilehto J, Hong Z, Cerebrolysin Acute Stroke Treatment in Asia (CASTA) Investigators. Cerebrolysin in patients with acute ischemic stroke in Asia: results of a double-blind, placebo-controlled randomized trial. Stroke. 2012;43:630-6.

23. Khalili H, Niakan A, Ghaffarpasand F. Effects of cerebrolysin on functional recovery in patients with severe disability after traumatic brain injury: a historical cohort study. Clin Neurol Neurosurg. 2017;152:34-8.

24. Yang Y, Zhang Y, Wang Z, Wang S, Gao M, Xu R, et al. Attenuation of acute phase injury in rat intracranial hemorrhage by cerebrolysin that inhibits brain edema and inflammatory response. Neurochem Res. 2016;41:748-57. 